\title{
Variability patterns of epibenthic microalgae in eastern Tunisian coasts
}

\author{
Malika Moncer 1,2, Asma Hamza 2, Wafa Feki-Sahnoun ${ }^{2}$, Lotfi Mabrouk ${ }^{3}$, Malika Bel Hassen ${ }^{4}$ \\ ${ }^{1}$ Faculté des Sciences de Sfax, BP 802, Route de la Soukra km 4, 3038 Sfax, Tunisia. \\ (MM) (Corresponding author) E-mail: malika.moncer@ hotmail.fr. ORCID iD: http://orcid.org/0000-0002-3344-6039 \\ ${ }_{2}^{2}$ Institut National des Sciences et des Technologie de la Mer de Sfax, Route Ibn Battouta, ZI Madagascar, 3000 Sfax, \\ Tunisia. \\ (AA) E-mail: asma.hamza@instm.rnrt.tn. ORCID iD: http://orcid.org/0000-0001-8228-012X \\ (WF-S) E-mail: wafafeki@yahoo.fr. ORCID iD: http:///orcid.org/0000-0003-1688-2071 \\ ${ }^{3}$ Faculté des Sciences de Gafsa, Campus Universitaire Sidi Ahmed Zarroug, 2112 Gafsa, Tunisia. \\ (LM) E-mail: lotfi2328@yahoo.fr. ORCID iD: http://orcid.org/0000-0001-8356-6058 \\ ${ }^{4}$ Institut National des Sciences et des Technologie de la Mer de Salammbô, 28 rue 2 mars 1934, 2025 Salammbô, Tunisia. \\ (MBH) E-mail: belhassen.malika@instm.rnt.tn. ORCID iD: http:// orcid.org/0000-0001-7412-9948
}

\begin{abstract}
Summary: Epiphytic microalgae were monitored on various substrates of seagrass and macroalgae and in the water column for one year (from March 2013 to March 2014) in Oued Lafrann along the eastern coast of Chebba (Tunisia) with a focus on the distribution patterns of the epibenthic toxic dinoflagellates Ostreopsis cf. ovata, Prorocentrum lima and Coolia monotis. Microalgae assemblages were dominated by diatoms and dinoflagellates both in the water column and on vegetation. High concentrations of epiphytic toxic and potentially toxic dinoflagellates were preferentially hosted by Posidonia leaves, mainly in the apical and middle regions of the leaves, and P. lima was the dominant species. A significant positive correlation was found between P. lima concentrations on Posidonia and in the water column, suggesting that macrophytes should be sampled in the framework of harmful algal species monitoring. Ostreopsis cf. ovata, exhibited low concentrations and was mainly present on the inner surface of the Posidonia leaf, whereas $P$. lima was mainly present on the outer surface of the leaf, suggesting a likely space competition.
\end{abstract}

Keywords: Prorocentrum lima; Ostreopsis cf. ovata; Coolia monotis; Prorocentrum micans; magnoliophytes; macroalgae.

\section{Patrones de variabilidad de las microalgas epibentónicas en la costa este tunecina}

Resumen: Las microalgas epifitas fueron monitorizadas en varios sustratos de praderas marinas, macroalgas y en la columna de agua durante un año (de marzo 2013 hasta marzo 2014) en Oued Lafrann, en la costa este de Chebba (Túnez), con especial atención a la distribución de dinoflagelados tóxicos epibénicos, como Ostreopsis cf. ovata, Prorocentrum lima y Coolia monotis. Las comunidades de microalgas estaban dominadas por diatomeas y dinoflagelados, tanto en la columna de agua como en la vegetación. Se encontraron elevadas concentraciones de dinoflagelados epífitos tóxicos preferentemente en las hojas de Posidonia, principalmente en las regiones apicales y media y Prorocentrum lima fue la especie epífita dominante. Se observó una correlación positiva significativa entre las concentraciones de $P$. lima en Posidonia y en la columna de agua, lo que sugiere el muestreo de esta macrófita como parte de la monitorización de especies nocivas. Las células de Ostreopsis cf. ovata mostraron concentraciones bajas y se encontraron principalmente en la superficie interior de la hoja de Posidonia contrariamente a la distribución de $P$. lima presente principalmente en la superficie exterior de la lámina. Este hecho probablemente sugiere una competencia espacial entre los dos microalgas tóxicas.

Palabras clave: Prorocentrum lima; Ostreopsis cf. ovata; Coolia monotis; Prorocentrum micans; magnoliophyta; macroalgas.

Citation/Como citar este artículo: Moncer M., Hamza A., Feki-Sahnoun W., Mabrouk L., Bel Hassen M. 2017. Variability patterns of epibenthic microalgae in eastern Tunisian coasts. Sci. Mar. 81(4): 487-498. doi: http://dx.doi.org/10.3989/scimar.04651.17A

Editor: E. Garcés.

Received: April 4, 2017. Accepted: September 21, 2017. Published: October 20, 2017.

Copyright: () 2017 CSIC. This is an open-access article distributed under the terms of the Creative Commons Attribution (CC-by) Spain 3.0 License. 


\section{INTRODUCTION}

Diverse and highly productive epiphytic assemblages composed mainly of microscopic algae are attached to the seagrass and macroalgae leave and benefit from this relationship by gaining a structure on which to grow and by consuming nutrients that the vegetation releases (Hauxwell et al. 2001, Perez et al. 2008).

The host plants play a key role in shaping the composition of the epiphytic community structure (Johnson et al. 2005), since the phenological parameters such as leaf length, leaf area and leaf area index of some magnoliophytes increase during the warm seasons (Mabrouk et al. 2009). The structure of epiphytic communities is also influenced by factors such as the age of the leaf (Mazzella et al. 1994), the seasonal cycle of macroalgae (Gambi et al. 1992) and grazing (Mirella et al. 2012).

The composition and abundance of epiphyte communities can also be influenced by abiotic factors such as irradiance, temperature, salinity and inorganic nutrients. Temperature contributes significantly to the temporal variation of diatom epiphytes (Johnson et al. 2005) and epiphytic dinoflagellates (Armi et al. 2010), and salinity is an important factor in the distribution of the microepiphyte community (Johnson et al. 2005). The influence of other factors such as hydrodynamics and light intensity on the development of epiphytes has also been documented (Nesti et al. 2009).

Epiphytic microalgae may include many toxic species that can damage fisheries and cause human health hazards. In recent years, the proliferation of toxic epibenthic species appears to be expanding on a global scale, probably due to either global climate change (Hallegraeff 2010) or anthropogenic impacts such as eutrophication and transfer of ballast water (Hallegraeff et al. 2010). The increase in studies in various ecosystems all around the world over the past few decades could also explain their apparent global proliferation
(Van Dolah 2000, Maso and Garcés 2006). Few studies (Bomber et al. 1989) have focused on the effect of the substrata on the growth of toxic epiphytic species, and their relationships with their hosts. Although the distribution of epiphytes has been shown to depend largely on their host (Cohu et al. 2013, Accoroni et al. 2016a), the question that still needs to be addressed is whether there is an affinity between a toxic species and a given substrate. Furthermore, Aligizaki and Nikolaidis (2006) highlighted correlations between the abundance of these toxic species in the water column and their abundance on macrophytes. Since most environmental monitoring programmes have focused on water column concentration of toxic species, understanding the distribution patterns of epiphytic species on macrophytes should be particularly useful for the design of monitoring programmes.

This study aims to characterize the temporal variability of epiphytic microalgae on different substrates (magnoliophytes and macroalgae) and in the water column, with a special focus on epiphytic toxic dinoflagellates. We particularly wish to examine the following hypotheses:

1) Do the diversity and abundance of epiphytic microalgae vary between substrata and environmental conditions?

2) Is there a relationship between the concentrations of the toxic species present on specific substrata and in the water column?

3) Do toxic epiphytic species show different distribution patterns on the leaves of Posidonia oceanica?

\section{MATERIALS AND METHODS}

\section{Study area}

The study area was in the locality of Oued Lafrann $\left(35^{\circ} 15^{\prime} 18^{\prime \prime} \mathrm{N}, 11^{\circ} 07^{\prime} 28^{\prime \prime} \mathrm{E}\right)$ in the region of Chebba (north of the Gulf of Gabès in Tunisia) (Fig. 1). The

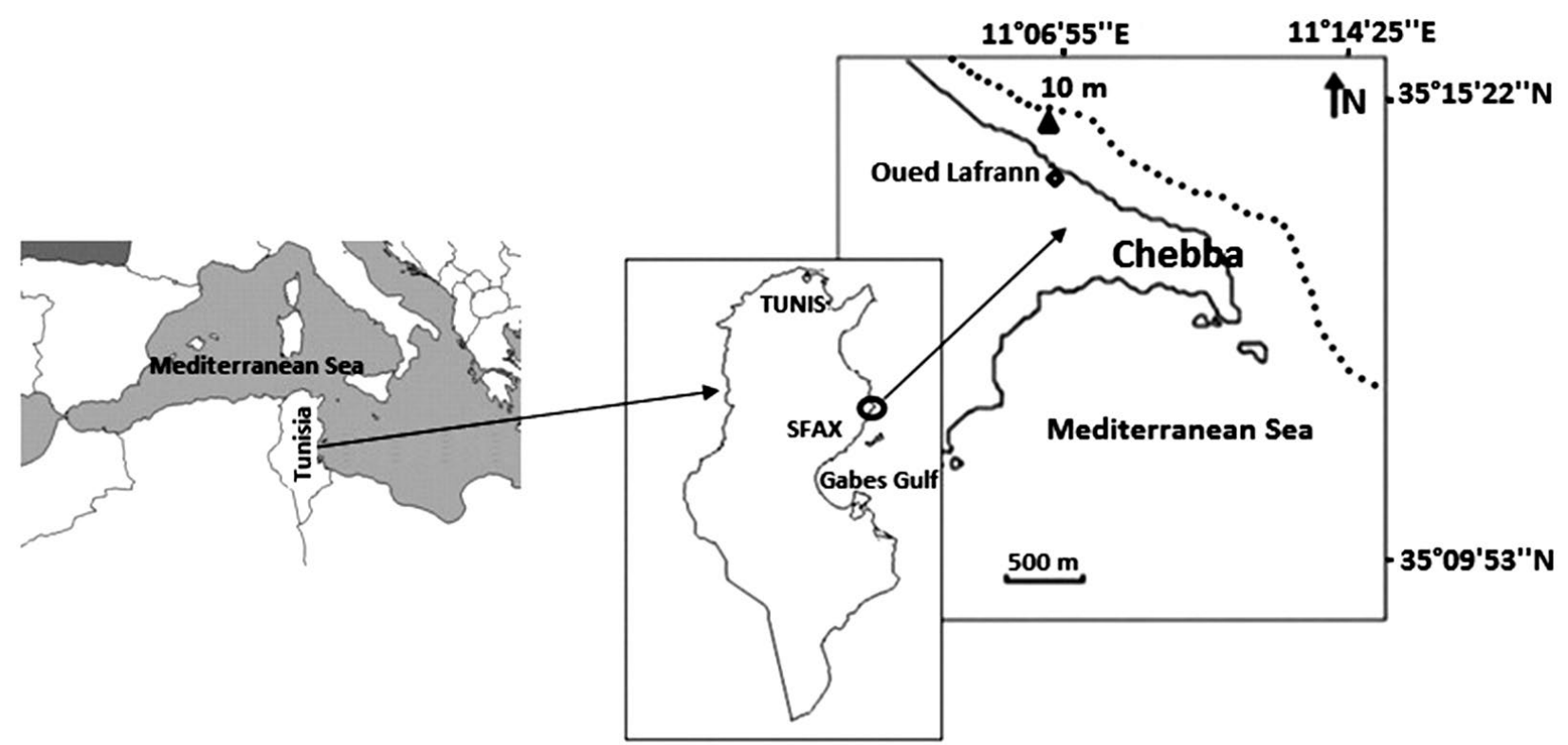

Fig. 1. - Map of the study area, showing the sampling station. 
climate is semiarid and sunny with strong northward winds. This region is not subjected to a major human impact. It has clear water in which artisanal and selective fisheries are very active.

The study area is colonized by many macrophytes. Cymodocea nodosa is present in shallow water (from $0.5 \mathrm{~m}$ to $18 \mathrm{~m}$ deep). P. oceanica beds, with foliage density exceeding 455.25 shoots $\mathrm{m}^{-2}$, follow Cymodocea and reach up to $20 \mathrm{~m}$ depth. The new invasive magnoliophyte Halophila stipulacea (Sghaier et al. 2011) with scattered tufts $\left(2-3 \mathrm{~m}^{2}\right)$ has also been recorded in this area. Chlorophyta Penicillus capitatus (Lamarck), with a density exceeding 1000 ind $\mathrm{m}^{-2}$, is intermixed with Cymodocea. Zostera noltii is identified in scattered tufts in shallow muddy hollows and is sometimes associated with the seagrass $C$. nodosa (Caye and Meinesz 1985). Photophylic algae generally colonize rocks, and tough substrates between 0.5 and 3 $\mathrm{m}$ depth, such as Cystoseira, represented by Cystoseira amentacea, Cystoseira stricta, Cystoseira compressa, Cystoseira barbata, occupy sandy bottoms.

\section{Data sampling and processing}

The sampling was conducted in a small creek covering a coastline of about $500 \mathrm{~m}$, where diverse substrates (rocky blocks and sandy surfaces with dense vegetation) were present. The sampling, performed monthly from March 2013 to March 2014 in the same creek, was conducted by diving from 0.5 to $2 \mathrm{~m}$ depth. The study area was well covered by different types of marine vegetation. Ten substrata were investigated: four magnoliophytes (Posidonia oceanica, Zostera noltii, Cymodocea nodosa and Halophila stipulacea) and six macroalgae (Padina pavonica, Cystoseira mediterranea, Dictyota dichotoma, Dictyopteris membranacea, Penicilus capitatus, Asparagopsis armata). Most of the sampled vegetation was not permanent during the sampling period. Some types of vegetation, such as Posidonia oceanica, were present throughout the year; others such as Padina pavonica, Cystoseira mediterranea and Halophila stipulacea, appeared for a few months. $P$. oceanica, a perennial species, showed a rather good vitality in the study area, as was confirmed by several previous studies (Mabrouk et al. 2009, 2011).

Macrophytes and seawater samples were collected in triplicate following the protocol agreed by a consortium of experts in the framework of the ENPICBCMED project M3-HABs (http://m3-habs.net) and recently published in Accoroni et al. (2016b). Before collection of the benthic substrata and in order to avoid resuspension, we sampled $1.5 \mathrm{~L}$ of seawater at about $30 \mathrm{~cm}$ from the macrophyte in plastic bottles for nutrient analysis $(500 \mathrm{ml})$ and planktonic identification $(1 \mathrm{~L})$. The leaf beam of the magnoliophytes and total macroalgal thalli were then covered with a plastic bag (two different sizes, 50/30 cm and 40/20 $\mathrm{cm}$, depending on the size of the vegetation) and gently detached from their substrate. The plant samples within the storage water were shaken vigorously to dislodge the epiphytic cells. They were then re-rinsed with filtered sea water (FSW) (2x $100 \mathrm{ml})$. The total retrieved volume was noted. It generally ranged between 400 and $1000 \mathrm{ml}$.

The macrophyte was then weighed to determine the fresh weight. All collected samples were preserved in a seawater formalin (3\%o) solution and kept in the dark at ambient temperature until transfer to the laboratory.

For each retrieved sample, three subsamples (10 $\mathrm{mL}$ ) were counted by means of an inverted microscope according to Utermohl's sedimentation method (Utermohl 1958). The number of epiphyte species and their abundance, expressed as number of individuals per $g$ of fresh weight of macrophyte (FW), was determined for each sampling period and depth.

Some of the recorded dinoflagellates, namely $O s$ treopsis cf. ovata and P. lima, were reported to be toxic and others, such as $C$. monotis, to be potentially toxic (Calabretti et al. 2017, David et al. 2017). C. monotis strains collected in the Gulf of Gabès were shown to be toxic to mice after intra-peritoneal injection $\left(3.610^{7}\right.$ cells $\mathrm{ml}^{-1}$ ), causing loss of coordination, hind limb paralysis and respiratory difficulty (Abdennadher 2014).

During the period of confirmed high abundance of epiphytic toxic (Ostreopsis cf. ovata, Prorocentrum lima) and potentially toxic (Coolia monotis) dinoflagellates, generally occurring in September (Mabrouk et al. 2014), a triplicate of shoots (that totalized 15 leaf bundles) of $P$. oceanica were prospected in the densest meadows ( $2 \mathrm{~m}$ depths). The different sections of the leaf (apical, middle and basal) were separated in the field and each part was gently covered with a plastic bag. In the laboratory, two persons held the sectioned parts horizontally by two clamps on each side, and the inner and outer sides of the leaf were gently scraped with a lamella. The scrapings were immersed in $10 \mathrm{ml}$ of filtered sea water formalin (3\%o). The abundance of microepiphyte was expressed by cells $\mathrm{g}^{-1} \mathrm{FW}$ on each part of the leaf.

Water column temperature was measured in situ using a multi-parameter type 340i / SET. Inorganic nutrients $\left(\mathrm{NO}_{2}^{-}, \mathrm{NO}_{3}{ }^{-}, \mathrm{NH}_{4}{ }^{+}, \mathrm{PO}_{4}{ }^{3-}, \mathrm{Si}(\mathrm{OH})_{4}\right)$, totalnitrogen (TN) and total phosphate (TP) were analysed with a BRAN and LUEBBE type 3 autoanalyser, and concentrations were determined colorimetrically using a UV-visible (6400/6405) spectrophotometer (APHA 1992).

\section{Data analysis}

The Shannon index (Gray et al. 1992) was calculated to express diversity taking into account the number of species and abundance of individuals within each species. It is given by the following formula:

$$
H^{\prime}=-\sum_{i=1}^{S} p_{i} \log p_{i}
$$

where $p_{i}$ is the proportional abundance or percentage of the species importance: $p_{i}=n_{i} / N ; S$ is the total number of species; $n_{i}$ is the number of individuals of a species in the sample; $N$ is the total number of individuals of all species in the sample.

To examine the relationships between the abundance of toxic and potentially toxic epiphytic dinoflag- 
Table 1. - Temperature and nutrient concentrations (in $\mu \mathrm{mol} \mathrm{L}^{-1}$ ) expressed as the mean values $( \pm \mathrm{SD})$ of the samples taken during the study period. Values without SD correspond to a single record when only one sample was taken.

\begin{tabular}{|c|c|c|c|c|c|c|c|c|c|c|}
\hline Substrate & $\begin{array}{l}\text { Sample } \\
\text { number }\end{array}$ & $\begin{array}{l}\text { Depth } \\
\text { sample } \\
\text { (m) }\end{array}$ & $\mathrm{T}\left({ }^{\circ} \mathrm{C}\right)$ & $\mathrm{NO}_{2}^{-}$ & $\mathrm{Si}(\mathrm{OH})_{4}$ & $\mathrm{NO}_{3}^{-}$ & $\mathrm{NH}_{4}^{+}$ & $\mathrm{PO}_{4}^{3-}$ & $\mathrm{TN}$ & $\mathrm{TP}$ \\
\hline Posidonia oceanica (L.) Delile & 13 & 1.75 & $\begin{array}{l}18.06 \\
\pm 5.43\end{array}$ & $\begin{array}{l}0.966 \\
\pm 0.9\end{array}$ & $\begin{array}{l}3.341 \\
\pm 2.41\end{array}$ & $\begin{array}{l}7.181 \\
\pm 3.07\end{array}$ & $\begin{array}{l}6.274 \\
\pm 4.07\end{array}$ & $\begin{array}{l}2.892 \\
\pm 2.74\end{array}$ & $\begin{array}{l}23.286 \\
\pm 8.23\end{array}$ & $\begin{array}{l}12.478 \\
\pm 7.48\end{array}$ \\
\hline Padina pavonica Linnaeus & 7 & 1.8 & $\begin{array}{c}19.19 \\
\pm 7\end{array}$ & $\begin{array}{l}0.555 \\
\pm 0.13\end{array}$ & $\begin{array}{l}2.666 \\
\pm 2.21\end{array}$ & $\begin{array}{l}5.839 \\
\pm 1.43\end{array}$ & $\begin{array}{l}5.758 \\
\pm 5.21\end{array}$ & $\begin{array}{l}3.927 \\
\pm 3.36\end{array}$ & $\begin{array}{l}18.738 \\
\pm 7.12\end{array}$ & $\begin{array}{l}14.937 \\
\pm 8.76\end{array}$ \\
\hline $\begin{array}{l}\text { Cystoseira mediterranea } \\
\text { Sauvageau }\end{array}$ & 8 & 1.75 & $\begin{array}{l}16.73 \\
\pm 4.57\end{array}$ & $\begin{array}{l}1.054 \\
\pm 0.94\end{array}$ & $\begin{array}{l}3.138 \\
\pm 2.49\end{array}$ & $\begin{array}{l}7.065 \\
\pm 4.31\end{array}$ & $\begin{array}{l}5.703 \\
\pm 3.63\end{array}$ & $\begin{array}{l}2.178 \\
\pm 1.17\end{array}$ & $\begin{array}{l}23.653 \\
\pm 9.91\end{array}$ & $\begin{array}{l}11.490 \\
\pm 5.02\end{array}$ \\
\hline Halophila stipulacea Forsskål & 3 & 2 & $\begin{array}{l}14.03 \\
\pm 1.36\end{array}$ & $\begin{array}{l}0.887 \\
\pm 0.64\end{array}$ & $\begin{array}{l}2.764 \\
\pm 1.52\end{array}$ & $\begin{array}{l}7.143 \\
\pm 1.54\end{array}$ & $\begin{array}{c}9.401 \\
\pm 10.56\end{array}$ & $\begin{array}{l}1.461 \\
\pm 0.99\end{array}$ & $\begin{array}{l}25.453 \\
\pm 9.81\end{array}$ & $\begin{array}{l}8.436 \\
\pm 3.56\end{array}$ \\
\hline Dictyota dichotoma Hudson & 2 & 1.5 & $\begin{array}{l}12.4 \\
\pm 2.55\end{array}$ & $\begin{array}{l}0.405 \\
\pm 0.38\end{array}$ & $\begin{array}{l}3.470 \\
\pm 0.76\end{array}$ & $\begin{array}{l}2.638 \\
\pm 0.51\end{array}$ & $\begin{array}{l}17.075 \\
\pm 2.26\end{array}$ & $\begin{array}{l}2.992 \\
\pm 2.86\end{array}$ & $\begin{array}{l}27.357 \\
\pm 2.77\end{array}$ & $\begin{array}{l}15.361 \\
\pm 11.9\end{array}$ \\
\hline Zostera noltii Horneman & 1 & 2 & 14.2 & 0.301 & 1.918 & 5.030 & 2.795 & 0.506 & 17.427 & 4.994 \\
\hline Cymodocea nodosa Ucria & 1 & 2 & 24.2 & 0.482 & 2.286 & 5.112 & 3.56 & 3.215 & 14.982 & 13.762 \\
\hline Asparagopsis armata Harvey & 1 & 1 & 10.6 & 0.208 & 0.846 & 3.161 & 6.312 & 1.980 & 18.930 & 11.991 \\
\hline Penicillus capitatus Lamarck & 1 & 2 & 24.2 & 0.452 & 2.112 & 5.115 & 3.572 & 3.312 & 14.832 & 13.752 \\
\hline Dictyopteris membranacea Stackhouse & 1 & 2 & 24.6 & 0.612 & 7.215 & 4.672 & 17.433 & 11.12 & 32.412 & 33.109 \\
\hline
\end{tabular}

ellates on macrophyte leaves and in the water column, the bivariate Pearson correlation test was used (SPSS software).

One-way ANOVA analyses were conducted to test the difference in toxic epiphyte concentrations between the studied substrata and to compare the abundances of epiphytic toxic dinoflagellates on the different parts of $P$. oceanica leaves. The Student-Newman-Keuls (SNK) post hoc test was used for post hoc multiple comparisons of means (Underwood 1997). Cochran's $\mathrm{C}$ test was used before each analysis to check the homogeneity variance and data were $\log (\mathrm{x}+1)$ transformed when necessary (Underwood 1997).

The similarity in epiphytic composition and abundance between the studied substrates was analysed by means of cluster analyses. We conducted a hierarchical agglomerative clustering analysis (Clarke and Warwick 2001) using the routine "CLUSTER" of the PAST software to depict the relative differences in epiphytic substrata.

A co-inertia analysis (Dolédec and Chessel 1994), which is a direct extension of multiple regressions to the modelling of a multivariate response matrix (Legendre and Legendre 1998), was conducted to examine the correlation between an array of response variables (in this case the ten substrates) and of independent explanatory variables (epibenthic abundance) conditional to a third matrix (here environmental parameters), keeping the environmental effect constant. A simple $\log (\mathrm{x}+1)$ transformation was applied to the data to stabilize variance (Frontier 1973). Computing and graphical displays were performed with R-2.4.0 software (R-Development Core Team 2006) using the packages ade4 1.4.2 (Chessel et al. 2012).

\section{RESULTS}

Detailed nutrient mean concentrations are reported in Table 1. These mean concentrations were calculated taking into account the number of samples of each substrate during the study period. The results showed that nutrient concentrations varied from one substrate to another. Zostera noltii and Asparagopsis armata exhibited more variability than the other substrates.
The concentrations of nitrite and nitrate were high in magnoliophytes, with a maximum recorded for $P$. oceanica, whereas in macroalgae the maximum was recorded for Cystoseira mediterranea. Ammonium concentrations showed a different trend, with the highest values being recorded in macroalgae (Table 1). The highest concentrations of phosphorus and silicate were recorded for Dictyopteris membranacea (Table 1).

During the study period, a total of 31 microalgal taxa were identified (Table 2). Three algal groups were represented in our study area: namely, Baccillariophyceae (19 species), Dinophyceae (9 species) and Cyanophyceae (3 species). The species number differed according to the host species (Table 2). The highest species diversity was recorded on $P$. oceanica and $A$. armata. Only a few species were recorded on macrophytes (Table 2).

On magnoliophytes, the highest abundances of diatoms were recorded on $P$. oceanica leaves during spring. This class was also rather important in spring and summer, with abundance exceeding $60 \%$ and reaching over $96 \%$ of total epiphyte microalgae on macroalgae, especially on Asparagopsis armata (Table 3).

Dinoflagellates accounted for the highest abundances on $P$. oceanica in winter. This class also showed high abundances on Cymodocea nodosa (Table 3). On macroalgae, Cystoseira mediterraneas howed the highest abundances of dinoflagellates. Comparing to diatoms, dinoflagellates showed a low abundance on Padina pavonica and this group was almost absent on Asparagopsis armata.

The diversity index $\left(H^{\prime}\right)$ of epibenthic species was very high on $P$. oceanica, $P$. pavonica, $H$. stipulacea and $D$. dichotoma (Table 3 ). The highest diversity index $\left(\mathrm{H}^{\prime}\right)$ was recorded on $P$. oceanica for magnoliophyte $\left(\mathrm{H}^{\prime}=3.656\right)$ and on $D$. dichotoma for macroalgae $\left(H^{\prime}=3.835\right)$ during spring, and the lowest was recorded on $Z$. noltii during the same season $\left(H^{\prime}=1.825\right)$ (Table 3$)$.

The co-inertia plot (Fig. 2A) illustrated close relationships between the composition of phytoplankton communities and the water properties above the ten sampling substrates. The overall model explained 33\% of the total variation (permutation test, $\mathrm{p}=0.02,1000$ replicates). This variation was due to microphytoplank- 
Table 2. - List of the counted species and the mean abundance (ind. $\left.\mathrm{g}^{-1} \mathrm{FW}\right)$ of microepiphytes in the locality of Chebba $(*, 0$; **, $<100$; ***, $101-500 ; * * * *, 501-1000 ; * * * * *,>1000)$.

\begin{tabular}{|c|c|c|c|c|c|c|c|c|c|c|}
\hline Supports & 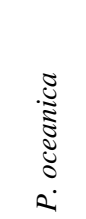 & 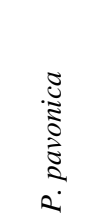 & 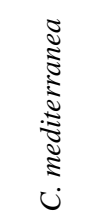 & 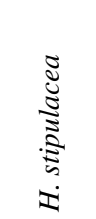 & 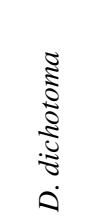 & $\begin{array}{c}: \mathbb{N} \\
\underset{\Xi}{\Xi} \\
N\end{array}$ & 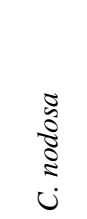 & 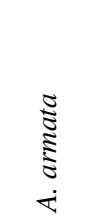 & 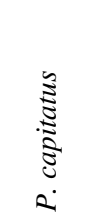 & 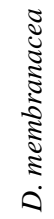 \\
\hline \multicolumn{11}{|l|}{ Dinophyceae } \\
\hline Prorocentrum lima & $* * * * *$ & $* * *$ & $* * * * *$ & $* * * *$ & $* * * *$ & $* * * *$ & $* * * *$ & $* * * * *$ & $* * * * *$ & $* *$ \\
\hline Ostreopsis cf. ovata & $* * *$ & $* *$ & $* *$ & $*$ & $*$ & $*$ & $* * *$ & $*$ & $*$ & $*$ \\
\hline Coolia monotis & $* * *$ & $* *$ & $* *$ & $* * *$ & $* * * *$ & $* * *$ & $* * *$ & $* * * *$ & $* * *$ & $* *$ \\
\hline Prorocentrum micans & $* *$ & $* *$ & $* *$ & $*$ & $* *$ & $*$ & $*$ & $*$ & $* *$ & $*$ \\
\hline Amphidinium sp. & $*$ & $*$ & $*$ & $*$ & $*$ & $*$ & $*$ & $*$ & $*$ & $*$ \\
\hline Polykrikos kofoidii & $* * * * *$ & $* * *$ & $* * *$ & $* * *$ & $* * *$ & $* * * * *$ & $* * * *$ & $* * * * *$ & $* * * * *$ & $* * *$ \\
\hline Peridinium sp. & $*$ & $*$ & $*$ & $*$ & $*$ & $*$ & $*$ & $*$ & $*$ & $*$ \\
\hline Alexandrium minitum & * & $*$ & $*$ & $*$ & * & $*$ & * & $*$ & $*$ & $*$ \\
\hline Protoperidinium sp. & $*$ & $*$ & $*$ & $*$ & $*$ & $*$ & $*$ & $*$ & $*$ & $*$ \\
\hline \multicolumn{11}{|l|}{ Baccilariophyceae } \\
\hline Navicula sp. & $* * * * *$ & $* * * * *$ & $* * * * *$ & $* * * * *$ & $* * * * *$ & $* * * *$ & $* * * * *$ & $* * * * *$ & $* * * * *$ & $* * *$ \\
\hline Navicula shmidtii Largerst & $* * * * *$ & $* * *$ & $* * *$ & $* * *$ & $* *$ & $* * *$ & $*$ & $* * * * *$ & $* * * *$ & $*$ \\
\hline Navicula gracilis Ehrenberg & $* * * *$ & $* *$ & $*$ & $* * * *$ & $* * *$ & $*$ & $* * *$ & $* * * * *$ & $* * *$ & $*$ \\
\hline Licmophora abbreviata C.Agardh & $* * * *$ & $* *$ & $*$ & $* * * *$ & $* * *$ & $* *$ & $* *$ & $* * * * *$ & $* * *$ & $* *$ \\
\hline Coscinodiscus concinnus W. Smith & $* * *$ & $* *$ & $* *$ & $* * *$ & $*$ & $*$ & $*$ & $* * * *$ & $*$ & $*$ \\
\hline Nitzschia sp. & $* * * * *$ & $* * * * *$ & $* * * * *$ & $* * * * *$ & $* * * * *$ & $* * *$ & $* * *$ & $* * * * *$ & $* * * *$ & $* *$ \\
\hline Pleurosigma sp. & $* * *$ & $* *$ & ** & $* * *$ & $* * *$ & $*$ & $* * *$ & $* * * * *$ & $* *$ & $* *$ \\
\hline Amphiprora sp. & $* * *$ & $*$ & $* * *$ & $* * *$ & $* * *$ & $* * *$ & $* *$ & $* * * * *$ & $* * *$ & $* *$ \\
\hline Amphora marina $\mathrm{W}$. Smith & $* * * *$ & $* *$ & $* * *$ & $* * * *$ & $* * *$ & $* * * *$ & $* *$ & $* * * * *$ & $* *$ & $* *$ \\
\hline Pinnularia viridis (Nitzsch) Ehrenberg & $* * *$ & * & $*$ & $* *$ & $*$ & $* *$ & $* *$ & $* * * *$ & * & $* *$ \\
\hline Achnanthes brevipes C. Agardh & $* * *$ & $* * *$ & $* *$ & $* *$ & $* * *$ & $* * *$ & $* *$ & $* * * * *$ & $*$ & $*$ \\
\hline Biddulphia sp. & $* *$ & $*$ & $*$ & $*$ & $* *$ & $*$ & $*$ & $* * *$ & $*$ & $*$ \\
\hline Chaetoceros sp. & $* *$ & $*$ & $*$ & $* *$ & $* *$ & $*$ & * & $* * *$ & * & $*$ \\
\hline Grammatophora sp. & $* * *$ & $* *$ & $*$ & $* *$ & $* * *$ & $* *$ & $*$ & $* * * * *$ & $* *$ & $*$ \\
\hline Gyrosigmaacuminatum (Kütz) Rabenh. & $* * *$ & $*$ & $* *$ & $* * *$ & $* * * *$ & $* *$ & $* *$ & $* * * * *$ & $* * *$ & $*$ \\
\hline Plagiotropis sp. & $* *$ & $*$ & $*$ & $*$ & $* *$ & $*$ & $*$ & $* * * *$ & $*$ & $*$ \\
\hline Skeletonema costatum & $* * *$ & $*$ & $*$ & $* *$ & $*$ & $*$ & $* *$ & $* * * *$ & $* *$ & $*$ \\
\hline Striatella unipunctata (Lyngbye) C.Agardh & $* * *$ & * & $*$ & $* *$ & $*$ & $* *$ & $*$ & $* * *$ & * & $*$ \\
\hline Thalassiosira aestivalis Gran & $* *$ & $* *$ & $*$ & $* *$ & $*$ & $* *$ & * & $* * *$ & $* *$ & $*$ \\
\hline \multicolumn{11}{|l|}{ Cyanophyceae } \\
\hline Anabaena sp. & $* * * *$ & $* * *$ & $* * *$ & $* * *$ & $* * * *$ & $* * *$ & $* *$ & * & $* * *$ & $* *$ \\
\hline Merismopedia sp. & $* * *$ & $*$ & $*$ & $* *$ & $* * * *$ & $* *$ & $* * *$ & $* * *$ & $* *$ & $*$ \\
\hline Oscillatoria sp. & $* * * *$ & $*$ & $* *$ & $*$ & $* * * * *$ & $* *$ & $*$ & $* *$ & $* * *$ & $*$ \\
\hline
\end{tabular}

Table 3. - Absolute abundance and seasonal percentages (\%) of abundance of different phytoplankton groups (relative to the total of epiphyte microalgae) sampled on various substrates. AA, absolute abundance (cells $\mathrm{g}^{-1} \mathrm{FW}$ ); $\mathrm{SD}$, standard deviation; $H^{\prime}$, diversity index.

\begin{tabular}{|c|c|c|c|c|c|c|c|c|c|}
\hline \multirow{2}{*}{ Substrate } & \multirow{2}{*}{ Season } & \multirow{2}{*}{ Diatoms } & \multicolumn{2}{|c|}{ Dinoflagellates } & \multirow{2}{*}{ Others } & \multirow{2}{*}{$H^{\prime}$} & \multirow{2}{*}{$\begin{array}{c}\text { Diatoms } \\
\text { AA }( \pm S D)\end{array}$} & \multirow{2}{*}{$\begin{array}{c}\text { Dinoflagellates } \\
\text { AA }( \pm S D)\end{array}$} & \multirow{2}{*}{$\begin{array}{c}\text { Others } \\
\text { AA }( \pm S D)\end{array}$} \\
\hline & & & Toxic & Non toxic & & & & & \\
\hline \multirow{4}{*}{ P. oceanica } & Spring & $61.75 \pm 1.43$ & $30.87 \pm 0.66$ & $3.69 \pm 0$ & 3.69 & 3.656 & $50200 \pm 5374$ & $28100 \pm 4313$ & $3000 \pm 565$ \\
\hline & Summer & $22.48 \pm 2.58$ & $60.74 \pm 0.64$ & $11.74 \pm 4.42$ & $5.03 \pm 1.19$ & 3.008 & $6700 \pm 1555$ & $21600 \pm 2121$ & $1500 \pm 495$ \\
\hline & Autumn & $39.64 \pm 1.96$ & $38.66 \pm 0.76$ & $3.94 \pm 0.37$ & $17.75 \pm 3.08$ & 3.205 & $20100 \pm 777$ & $21600 \pm 1272$ & $9000 \pm 2121$ \\
\hline & Winter & $13.11 \pm 3.26$ & $65.57 \pm 1.23$ & $16.39 \pm 6.46$ & $4.92 \pm 1.97$ & 2.566 & $800 \pm 282$ & $5000 \pm 565$ & $300 \pm 141$ \\
\hline \multirow{2}{*}{ P. pavonica } & Spring & $65.31 \pm 7.61$ & $20.41 \pm 4.79$ & 0 & $14.29 \pm 3.67$ & 2.120 & $1600 \pm 282$ & $500 \pm 70$ & $350 \pm 106$ \\
\hline & Autumn & $65.93 \pm 3.62$ & $24.18 \pm 2.02$ & $8.79 \pm 1.43$ & $1.1 \pm 0.18$ & 3.785 & $6000 \pm 1060$ & $3000 \pm 141$ & 100 \\
\hline \multirow{2}{*}{ C. mediterranea } & Spring & $49.34 \pm 11.32$ & $31.58 \pm 3.47$ & $13.16 \pm 5.77$ & $5.92 \pm 1.83$ & 3.503 & $7500 \pm 1060$ & $6800 \pm 777$ & $900 \pm 212$ \\
\hline & Spring & $64.52 \pm 1.43$ & $24.19 \pm 3.29$ & $8.06 \pm 2.3$ & $3.23 \pm 0.44$ & 3.738 & $8000 \pm 1060$ & $4000 \pm 353$ & 400 \\
\hline H. stipulacea & Winter & $65.98 \pm 3.69$ & $20.62 \pm 7.8$ & $10.31 \pm 4.6$ & $3.09 \pm 0.5$ & 3.236 & $6400 \pm 1131$ & $3000 \pm 141$ & 300 \\
\hline \multirow{2}{*}{$\begin{array}{l}\text { D. dichotoma } \\
\text { D. membranacea }\end{array}$} & Spring & $65.22 \pm 3.16$ & $16.67 \pm 1.43$ & $3.62 \pm 1.4$ & $14.49 \pm 0.33$ & 3.835 & $9000 \pm 1767$ & $2800 \pm 141$ & $2000 \pm 282$ \\
\hline & Summer & 50 & $25 \pm 5.89$ & $12.5 \pm 2.95$ & $12.5 \pm 2.95$ & 2.828 & $400 \pm 71$ & $300 \pm 71$ & 100 \\
\hline \multirow{2}{*}{$\begin{array}{l}\text { C. nodosa } \\
\text { Z. noltii }\end{array}$} & Autumn & $57.14 \pm 0.81$ & $28.57 \pm 1.01$ & $10.71 \pm 0.51$ & $3.57 \pm 0.3$ & 3.2 & $3200 \pm 283$ & $2200 \pm 141$ & 200 \\
\hline & Spring & $64.52 \pm 2.11$ & $24.19 \pm 3.51$ & $1.61 \pm 4.3$ & $9.68 \pm 1.32$ & 1.825 & $4000 \pm 566$ & $1600 \pm 141$ & 600 \\
\hline A. armata & Spring & $96.67 \pm 0.23$ & $1.16 \pm 0.11$ & $0.5 \pm 0.17$ & $1.66 \pm 0.18$ & 2.042 & $232500 \pm 8839$ & $4000 \pm 283$ & $4000 \pm 566$ \\
\hline P. capitatus & Autumn & $51.43 \pm 0.13$ & $22.86 \pm 4.37$ & $17.14 \pm 3$ & $8.57 \pm 1.5$ & 2.507 & $3600 \pm 283$ & $2800 \pm 141$ & $600 \pm 141$ \\
\hline
\end{tabular}

ton taxa (20\%) and to physical and chemical variability (18.52\%) (Fig. 2B). Posidonia, Cystoseira and Halophila substrates showed close links between nitrite and nitrate and the phytoplankton species, as was illustrated by the position of Ostreopsis, P. lima and total dinoflagellates (Fig. 2A). In contrast, Dictyota dichotoma, Zostera noltii and Asparagopsis armata substrates were surrounded by the numerically dominant Coolia monotis, total phytoplankton and diatoms (Fig. 2A).
The toxic and potentially toxic dinoflagellates were mostly concentrated on $P$. oceanica, where they represented about $65 \%$ of the total epiphyte microalgae, followed by Cystoseira mediterranea and Cymodocea nodosa.

On $P$. oceanica, the occurrence frequency of toxic and potentially toxic dinoflagellates was high both on substrate and in the water column (Table 4). The highest occurrence of toxic dinoflagellates was observed 
A

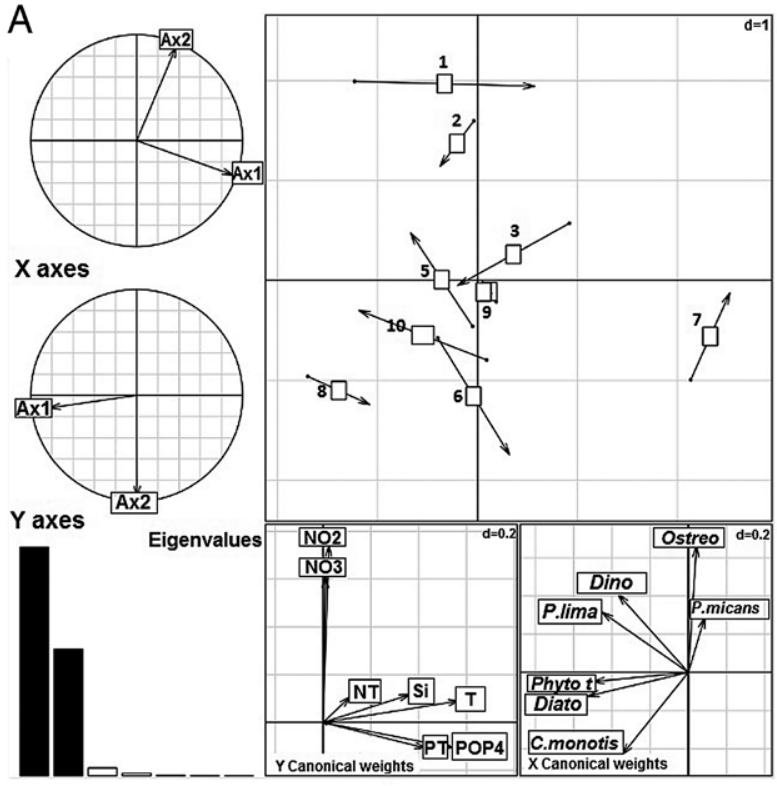

B

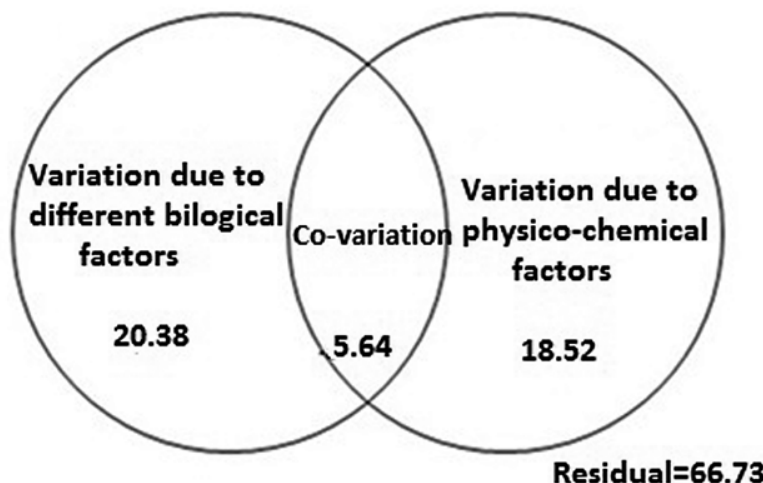

Fig. 2. - Co-inertia analysis of relationships between the relative abundance of epiphytic algae, substrates and environmental variable. 1, P. oceanica; 2, C. mediterranea; $3, P$. pavonica; 4 , C. nodosa; 5 , H. stipulacea; $6, D$. dichotoma; 7 , D. membranacea; $8, A$. armata; 9, P. capitatus; 10, Z. noltii; Dino, dinoflagellates; Diato, diatoms; Ostreo, Ostreopsis cf. ovata; Phy t, total phytoplankton.

Table 4. - Occurrence frequency of dinoflagellates (relative to total dinoflagellates) on P. oceanica and in its water column and their ecological characteristics (*, toxic; **, potentially toxic; a,be, according to Abdennadher (2014); ${ }^{\text {d }}$, according to Pagliara and Caroppo (2012); c, according to Calabretti et al. (2017), David et al. (2017), Abdennadher (2014).

\begin{tabular}{|c|c|c|c|c|c|c|}
\hline \multirow{2}{*}{ Species } & \multicolumn{2}{|c|}{ Biotope } & \multicolumn{2}{|c|}{ Occurrence frequency $(\%)$} & \multicolumn{2}{|c|}{ Maximum concentrations } \\
\hline & Benthic & Planktonic & Water column & Epiphytes & Cells L $\mathrm{L}^{-1}$ & Cells $\mathrm{g}^{-1} \mathrm{FW}$ \\
\hline P. lima *a & + & + & 54.26 & 66.83 & 2000 & 24300 \\
\hline Ostreopsis cf. ovata $* \mathrm{~b}$ & + & + & 9.30 & 1.84 & 300 & 2000 \\
\hline Coolia monotis $* * \mathrm{c}$ & + & + & 6.20 & 2.76 & 200 & 3100 \\
\hline P. micans & + & + & 0.78 & 0.81 & 100 & 1000 \\
\hline Amphidinium sp.*d & - & + & 1.55 & 0 & 100 & 0 \\
\hline Polykrikos kofoidii & + & + & 24.81 & 27.76 & 500 & 14600 \\
\hline Peridinium sp. & - & + & 0.78 & 0 & 100 & 0 \\
\hline Alexandrium minitum ${ }^{* \mathrm{e}}$ & - & + & 1.55 & 0 & 100 & 0 \\
\hline Protoperidinium sp. & - & + & 0.78 & 0 & 100 & 0 \\
\hline
\end{tabular}

Table 5. - Mean abundance (relative to total dinoflagellates) and seasonal percentages (\%) (relative to total epiphyte microalgae) of the epiphytic toxic dinoflagellates sampled on various substrates. MA, mean abundance.

\begin{tabular}{|c|c|c|c|c|c|c|c|c|c|}
\hline \multirow[b]{2}{*}{ Substrates } & \multirow[b]{2}{*}{ Season } & \multicolumn{2}{|c|}{ Ostreopsis cf. ovata } & \multicolumn{2}{|c|}{ Prorocentrum lima } & \multicolumn{2}{|c|}{ Coolia monotis } & \multicolumn{2}{|c|}{ Prorocentrum micans } \\
\hline & & MA & $\%$ & MA & $\%$ & MA & $\%$ & MA & $\%$ \\
\hline \multirow[t]{4}{*}{ P. oceanica } & Spring & $63 \pm 12$ & $0.22 \pm 0.03$ & $17588 \pm 521$ & $62.59 \pm 1.57$ & $925 \pm 88$ & $3.29 \pm 0.15$ & $75 \pm 11$ & $0.27 \pm 0.03$ \\
\hline & Summer & $50 \pm 14$ & $0.23 \pm 0.05$ & $6233 \pm 731$ & $28.86 \pm 0.53$ & $150 \pm 35$ & $0.69 \pm 0.11$ & $17 \pm 5$ & $0.08 \pm 0.02$ \\
\hline & Autumn & $217 \pm 12$ & 1 & $383 \pm 45$ & $1.77 \pm 0.1$ & $33 \pm 9$ & 0.15 & 0 & 0 \\
\hline & Winter & $83 \pm 14$ & $1.67 \pm 0.1$ & $2050 \pm 318$ & $41 \pm 0.7$ & 0 & 0 & $300 \pm 71$ & $6 \pm 0.7$ \\
\hline \multirow[t]{2}{*}{ P. pavonica } & Spring & 0 & 0 & $400 \pm 71$ & $80 \pm 3.54$ & $50 \pm 35$ & $10 \pm 7.07$ & 0 & 0 \\
\hline & Autumn & 67 & 2.22 & $617 \pm 70$ & $20.56 \pm 2.08$ & 100 & $3.33 \pm 0.86$ & $50 \pm 35$ & $1.67 \pm 1.18$ \\
\hline C. mediterranea & Spring & 0 & 0 & $4325 \pm 477$ & $63.6 \pm 4.66$ & $175 \pm 39$ & $2.57 \pm 0.19$ & $125 \pm 28$ & $1.84 \pm 0.14$ \\
\hline \multirow[t]{2}{*}{ H. stipulacea } & Spring & 0 & 0 & $650 \pm 35$ & $16.25 \pm 0.63$ & $500 \pm 141$ & $12.5 \pm 2.78$ & 0 & 0 \\
\hline & Winter & 0 & 0 & $1025 \pm 124$ & $34.17 \pm 5.89$ & 50 & $1.67 \pm 0.59$ & 0 & 0 \\
\hline D. dichotoma & Spring & 0 & 0 & $1050 \pm 177$ & $37.5 \pm 0.8$ & $1050 \pm 71$ & $37.5 \pm 4.02$ & 50 & $1.79 \pm 0.34$ \\
\hline D. membranacea & Summer & 0 & 0 & $50 \pm 35$ & $16.67 \pm 5.89$ & $50 \pm 35$ & $16.67 \pm 11.79$ & 0 & 0 \\
\hline C. nodosa & Autumn & $500 \pm 71$ & $22.73 \pm 0.36$ & $700 \pm 71$ & $31.82 \pm 1.07$ & $200 \pm 71$ & $9.09 \pm 2.5$ & 0 & 0 \\
\hline Z. noltii & Spring & 0 & 0 & $141 \pm 15$ & $8.81 \pm 2.25$ & $400 \pm 71$ & $25 \pm 3.54$ & 0 & 0 \\
\hline A. armata & Spring & 0 & 0 & $283 \pm 59$ & $7.07 \pm 0.58$ & $750 \pm 247$ & $18.75 \pm 4.42$ & 0 & 0 \\
\hline P. capitatus & Autumn & $100 \pm 71$ & $3.57 \pm 2.53$ & $1100 \pm 71$ & $39.29 \pm 11.5$ & $350 \pm 106$ & $12.5 \pm 0.98$ & 50 & $1.79 \pm 0.7$ \\
\hline
\end{tabular}

for P. lima, with $66.83 \%$ on macrophytes and $54.26 \%$ in the water column (Table 4). The frequency was low for Ostreopsis, with only $1.84 \%$ on macrophytes and $9.30 \%$ in the water column (Table 4). C. monotis did not exceed $10 \%$ in the water column and was about $2.76 \%$ on macrophytes (Table 4 ). The other epiphytic species were barely observed within the water column and on macrophyte leaves, except for the epiphytic Polykrikos kofoidii (27.76\%) (Table 4).

The abundance of the epiphytic dinoflagellates $O s$ treopsis cf. ovata was higher on magnoliophytes than on macroalgae, especially for Cymodocea nodosa, on which it reached $22.73 \%$ of the total dinoflagellates in autumn (Table 5). This toxic species did not show a 
A

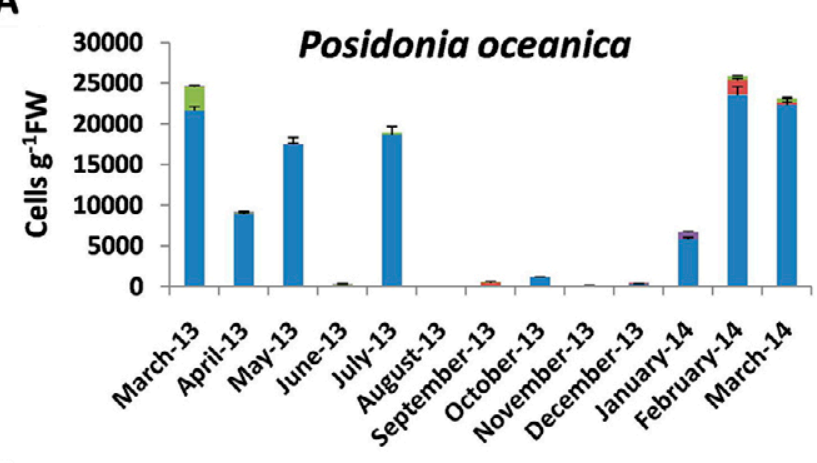

C

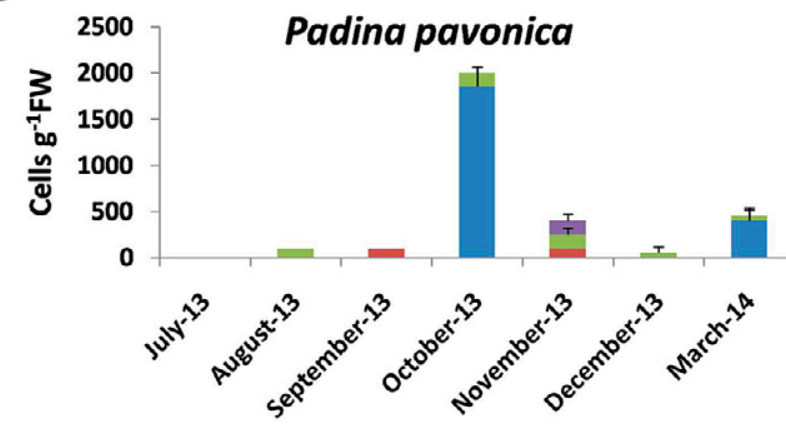

E

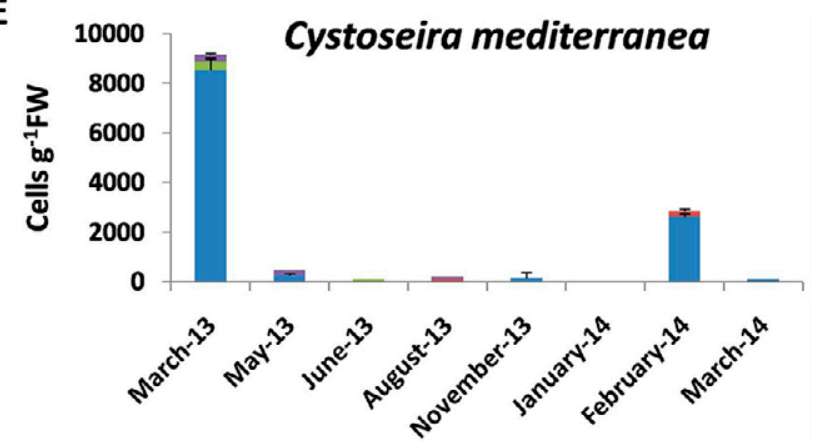

G

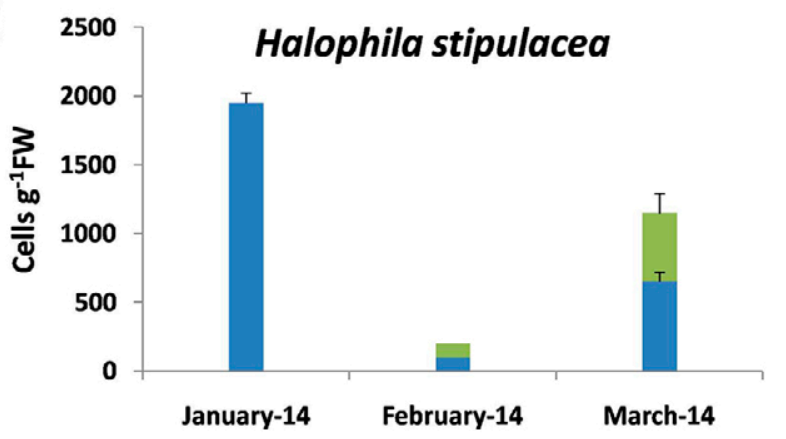

B

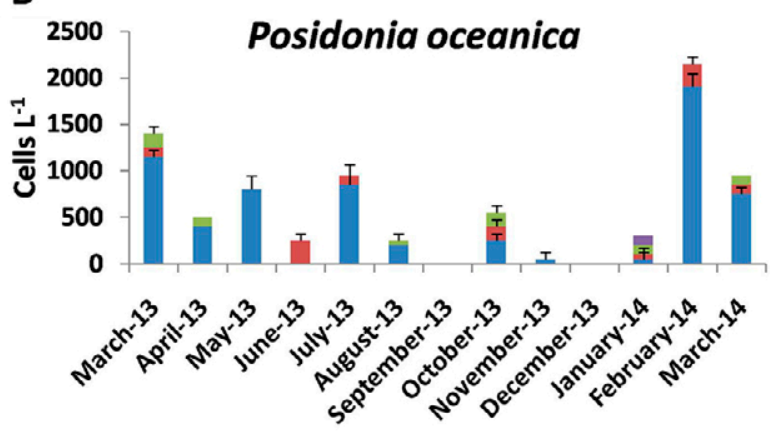

D

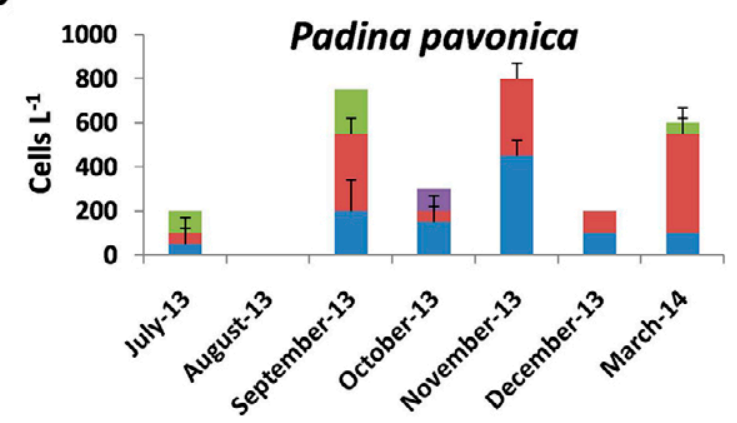

$\mathbf{F}$

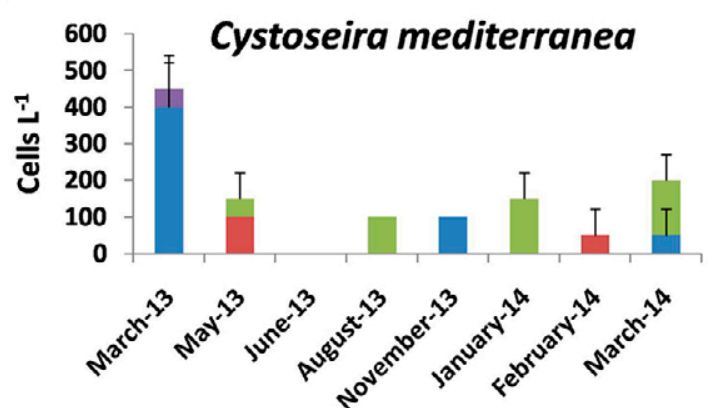

H

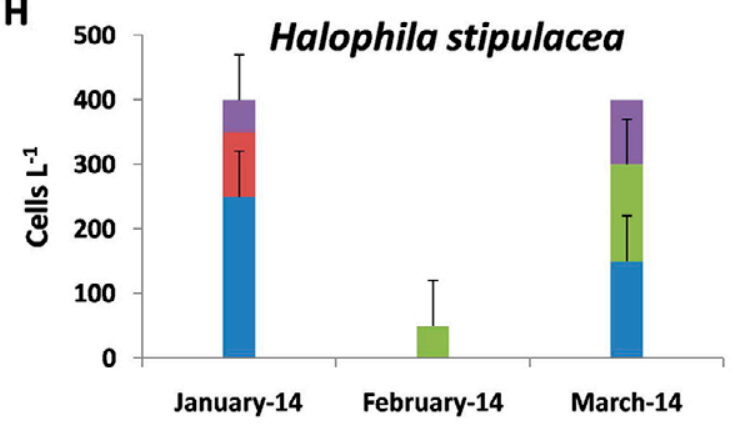

\section{$\backsim$ P. lima $\backsim$ O. cf. ovata $\backsim C$. monotis $\backsim$ P. micans}

Fig. 3. - Temporal distribution of epibenthic toxic dinoflagellates on the coasts of Chebba. Left, epiphytic dinoflagellates; right, dinoflagellates in water column.

significant difference in concentrations between the studied substrata, although the concentrations reached $0.510^{3}$ cells g ${ }^{-1} \mathrm{FW}$ on Cymodocea nodosa, $10^{3}$ cells $\mathrm{g}^{-1} \mathrm{FW}$ on Posidonia leaves in February and September (Fig. 3A), and relatively high abundances on Padina pavonica in September and November (Fig. 3C). A significant positive correlation $\left(\mathrm{P}<0.05, \quad \mathrm{R}^{2}=0.30\right)$ was observed between the species concentrations on
Padina pavonica and in the water column above this macroalga (Fig. 3C, D).

The abundance of $P$. lima on Posidonia leaves accounted for $62.59 \%$ of the total dinoflagellates (Table 5). During the sampling period, P. lima was the most dominant and frequent species on magnoliophytes as well as on macroalgae. This species significantly accumulated on $P$. oceanica $(\mathrm{P}<0.05)$, with the highest 


\begin{tabular}{lcccl|}
925 & 17588 & 63 & 75 & P. oceanica \\
50 & 400 & 0 & 0 & P. pavonica \\
1050 & 1050 & 0 & 50 & D. dichotoma \\
750 & 1700 & 0 & 0 & A. armata \\
500 & 650 & 0 & 0 & H. stipulacea \\
400 & 1000 & 0 & 0 & Z. noltii \\
117 & 2967 & 0 & 150 & C. mediterranea \\
\hline
\end{tabular}

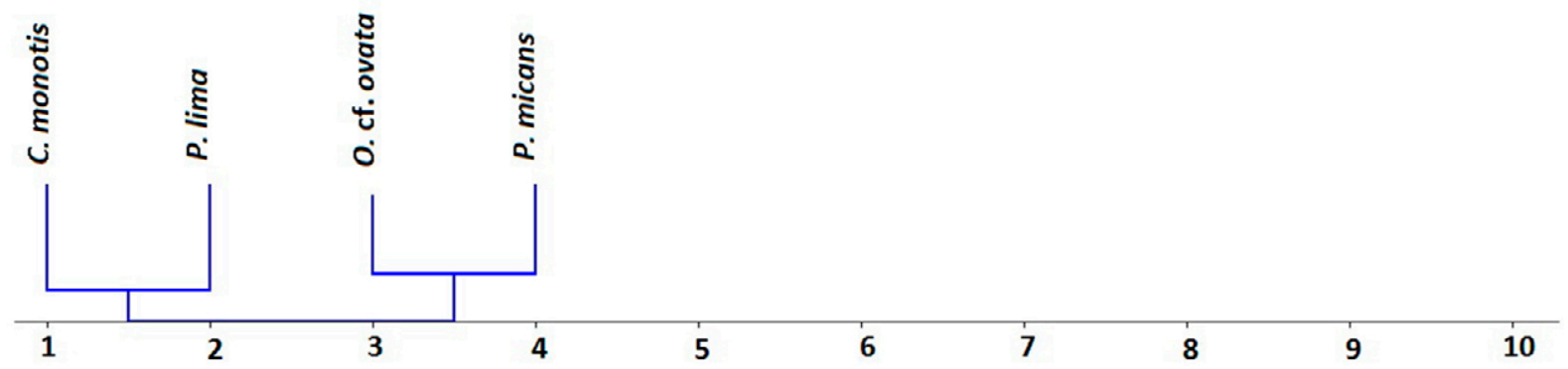

Fig. 4. - Degree of similarity between the different substrates in terms of spring average concentration of toxic dinoflagellates on each substrate.

concentrations, exceeding $10^{4}$ cells $\mathrm{g}^{-1} \mathrm{FW}$, being observed from February to May (Fig. 3A). On macroalgae, this species did not show significant variations between substrata. It exceeded $8.310^{3}$ cells $\mathrm{g}^{-1} \mathrm{FW}$ during March on Cystoseira mediterranea (Fig. 3E), whereas on Padina pavonica and on Halophila stipulacea, concentrations did not exceed $210^{3}$ cells g ${ }^{-1} \mathrm{FW}$ (Fig. 3C, G). In contrast to other toxic species, $P$. lima showed significant variations in its abundance on $P$. oceanica over time $(\mathrm{P}<0.05)$. This species was also present in the water column above the $P$. oceanica bed, with a concentration reaching over $10^{3}$ cells $\mathrm{L}^{-1}$ (Fig. $3 \mathrm{~B})$. A significant correlation $\left(\mathrm{P}<0.005, \mathrm{R}^{2}=0.80\right)$ was pointed out between $P$. lima concentrations on different substrata and in the water column.

C. monotis was also present on different substrates, with a maximum of $37.5 \%$ recorded on Dictyota dichotoma (Table 5). The monthly abundance of $C$. monotis showed no significant difference between the studied substrata $(\mathrm{P}>0.05)$ (Fig. 3). This species showed generally low concentrations in the water column sampled near Posidonia, Cystoseira and Halophila (Fig. 3F, H). $P$. micans showed the highest concentrations on $P$. oceanica but its abundances were rather low on other substrates (Table 5). Other substrata were barely observed in our study area. $P$. lima and $C$. monotis were the main species present on these substrates, where the maximum concentration was approximately $1.710^{3}$ cells $\mathrm{g}^{-1} \mathrm{FW}$ on Asparagopsis armata during April.
In spring, when the maximum of substrates were available, the clustering analysis of epiphytic species similarity between different substrates showed four groups (Fig. 4). The first cluster was composed of $C$. mediterranea, which hosted $C$. monotis, $P$. lima and $P$. micans (Fig. 4). The second cluster was composed of Z. noltii, H. stipulacea, A. armata and D. dichotoma, which hosted only two species, $P$. lima and $C$. monotis (Fig. 4). The third cluster was composed of $P$. pavonica, hosting mainly $P$. lima and $C$. monotis (Fig. 4). Finally, the last cluster was composed of $P$. oceanica, which showed high dissimilarity to the other substrates, hosting the different epiphytic dinoflagellates with a dominance of $P$. lima (Fig. 4).

According to the SNK test results, the distribution of Ostreopsis cf. ovata on Posidonia leaf revealed three groups (a homogeneous subset) (Table $6)$. The highest abundance was marked on the inner face of the apical and the middle parts of the leaf. This toxic species was also present with a relatively high abundance on the inner face of the basal part. On the other hand, it was particularly absent on the outer face of Posidonia leaf (Fig. 5). As regards $P$. lima, there were only two identified groups of the distribution of this species on the leaf of $P$. oceanica (Table 6). The first group was formed on the inner face of the middle part and the outer and inner face of the apical part, where the abundance was the highest (Fig. 5). The second group formed the outer

Table 6. - Student-Newman-Keuls test on the distribution of epibenthic toxic dinoflagellates on the leaf of Posidonia.

\begin{tabular}{|c|c|c|c|c|c|c|c|c|}
\hline \multirow[t]{2}{*}{ Face (O. cf. ovata) } & \multicolumn{3}{|c|}{$\begin{array}{l}\text { Subset for alpha }=0.05 \\
\text { (subgroups homogeneous of } \\
\text { averages which are not significantly } \\
\text { different from each other) }\end{array}$} & \multirow[t]{2}{*}{ Face $(P . \operatorname{lima})$} & \multicolumn{2}{|c|}{ Subset for alpha $=0.05$} & \multirow[t]{2}{*}{ Face (C. monotis) } & \multirow{2}{*}{$\begin{array}{c}\text { Subset for } \\
\text { alpha }=0.05 \\
1\end{array}$} \\
\hline & 1 & 2 & 3 & & 1 & 2 & & \\
\hline Apical part-outer face & .0000 & - & - & Middle part-outer face & 107.2000 & - & Middle part-inner face & .0000 \\
\hline Basal part-outer face & .0000 & - & - & Basal part-outer face & 128.4667 & - & Middle part-outer face & .0000 \\
\hline Middle part-outer face & 6.0667 & - & - & Basal part-inner face & 149.6667 & - & Basal part-outer face & .0000 \\
\hline Basal part-inner face & - & 103.6000 & - & Apical part-inner face & 199.8000 & 199.8000 & Apical part-outer face & 6.0667 \\
\hline Middle part-inner face & - & - & 196.0000 & Apical part-outer face & 225.6667 & 225.6667 & Apical part-inner face & 9.8667 \\
\hline Apical part-inner face & - & - & 201.8667 & Middle part-inner face & - & 311.8000 & Basal part-inner face & 21.6667 \\
\hline Significance & .976 & 1.000 & .840 & Significance & .120 & .063 & Significance & .372 \\
\hline
\end{tabular}




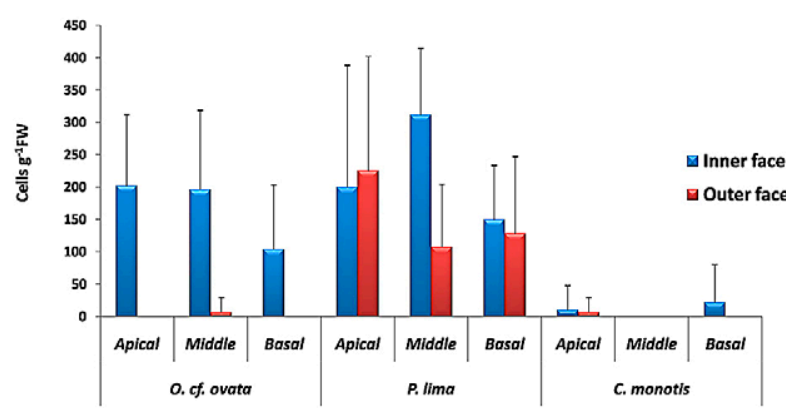

Fig. 5. - Distribution of epibenthic toxic dinoflagellates on the leaf of Posidonia oceanica.

Table 7. - One-way ANOVA result for abundances of epiphytic toxic dinoflagellates on the different parts of $P$. oceanica leaves, MS, mean square; F, Fisher test; p, significance level; in, inner face; ext, outer face, Ap, apical part of leaf; Ba, basal part; Mid, middle part; SNK, Student-Newman-Keuls; and ns, not significant.

\begin{tabular}{|c|c|c|c|c|c|}
\hline & Df & MS & $\mathrm{F}$ & $\mathrm{p}$ & SNK post hoc test \\
\hline \multicolumn{6}{|c|}{ Ostreopsis cf. ovata } \\
\hline Model & 5 & 5.514 & \multirow[t]{4}{*}{49.363} & \multirow[t]{4}{*}{$<0.0001$} & \multirow{4}{*}{$\begin{array}{c}\text { Mid in }=\text { Ap in }>\text { Ba in }> \\
\text { Mid ext }=\text { Ap ext }= \\
\text { Ba ext }\end{array}$} \\
\hline Residual & 84 & 0.112 & & & \\
\hline Total & 89 & & & & \\
\hline P. lima & & & & & \\
\hline Model & 5 & 0.869 & \multirow[t]{3}{*}{3.738} & \multirow[t]{3}{*}{0.004} & Mid in $=A p$ ext $=A p$ in $=$ \\
\hline Residual & 84 & 0.232 & & & $\mathrm{Ba}$ in $>$ Mid ext $=$ \\
\hline Total & 89 & & & & Ba ext \\
\hline \multicolumn{6}{|c|}{ C. monotis } \\
\hline Model & 5 & 0.065 & 1.106 & 0.363 & \multirow{3}{*}{ ns } \\
\hline Residual & 84 & 0.058 & & & \\
\hline Total & 89 & & & & \\
\hline
\end{tabular}

face of the middle and basal parts of the leaf, where the abundance was lower (Fig. 5).

The results of the ANOVA showed that the abundance of Ostreopsis cf. ovata and P. lima showed variability on the different parts of the Posidonia leaf (apical, middle and basal) (Fig. 5) and according to their position on the inner and outer faces of the leaves $\left(\mathrm{P}_{\text {Ostreopsis cf. ovata }}<0.0001 ; \quad \mathrm{P}_{\text {P.lima }}<0,005\right)$ (Table 7). Coolia monotis was only represented in a single subset that was recorded in low abundances and only on inner faces of Posidonia leaf (Fig. 5). The distribution showed no differences according to the face or part of the leaf $\left(\mathrm{P}_{\text {C.monotis }}>0.05\right)$ (Table 7).

\section{DISCUSSION}

Posidonia seagrass beds, in contrast to macrophytes, which are generally rather scattered with a high inter-annual variability, cover large areas of the Gulf of Gabès and are structured in valleys (Mabrouk et al. 2009, 2011, Ben Brahim 2013). During the year, Posidonia was by far the substrate hosting the greatest biomass and diversity of epiphytes (Table 3). This result could be explained by the diverse conditions that Posidonia offers for the success of epiphytic species: (i) the amount of physical structure usable as living space, as Posidonia provides both a shading effect and high microhabitat diversity because of its large leaf areas (Kikuchi and Pérès 1977); (ii) coexistence of Posidonia seagrass material, dead or alive, suspended particulate organic matter and leaf epiphytes as potential food sources within the ecosystem (Dauby 1989); (iii) protection from predators thanks to a dense rhizome mat; and (iv) the reduction of hydrodynamic forces (Lewis 1984). The P. oceanica canopy tends to mitigate currents and waves, thereby reducing the forces exerted on individual shoots (Koch et al. 2006).

Posidonia offers a greater surface for epiphytes than macroalgae such as Padina pavonica and Cystoseira mediterranea. However, the latter two host a relatively abundant population of epiphytes. Though they do not have the highest biomass of epiphytic species, marine macroalgae hosted the highest species diversity (Table 3 ), probably because they showed spatial complexity and could modulate the availability of resources, therefore affecting assemblages of associated epibiota (Gestoso et al. 2010). In particular, host algae with a branched structure like Cystoseira mediterranea or with a filamentous structure like Dictyota dichotoma usually have a high degree of structural complexity, which may make them more suitable as habitats for epibiota (Totti et al. 2009).

Diatoms were the dominant group and prevailed throughout the sampling period. This dominance could be attributed to their successful behaviour in attaching to the algae and establishing a mutualistic relationship with their host (Romagnoli et al. 2007). Indeed, pennate diatoms have the ability to cling to seaweeds by mucilage stalks and sheaths or gelatinous pads or by the attachment of the cell along its entire valve face. The centric forms are often trapped by the thallus of seaweeds or held in the tangle of attached forms (Totti et al. 2009).

A high diversity and abundance of confirmed toxic and potentially toxic dinoflagellate species hosted in vegetated habitats were recorded, especially on $P$. oceanica leaves (Table 3). Particularly P. lima, the most abundant species (Fig. 3), seems to affect $P$. oceanica leaves. This species has been reported as a widespread dinoflagellate in many coastal waters and estuaries around the world, generally in summer and autumn (Levasseur et al. 2003), in the Fleet lagoon in the UK (Foden et al. 2005), in Greek coastal waters (Aligizaki et al. 2009), along the coast and inside the harbours of the Abruzzo region in the Adriatic Sea (Ingarao et al. 2009), and on the northern coasts of Tunisia (Aissaoui et al. 2014). In the study area, it reached about 25000 cells $\mathrm{g}^{-1} \mathrm{FW}$ on Posidonia leaves, which is higher than the 70 cells $\mathrm{g}^{-1} \mathrm{FW}$ found in the same area by Mabrouk et al. (2011). However, these concentrations were lower than those reported for Cymodocea nodosa in Greece, where the abundance reached 133000 cells g-1 FW (Aligizaki et al. 2009). The high concentrations of P. lima on Posidonia raise the problem of its sampling representativeness, since most monitoring programmes focused on the water column, which might lead to an underestimation of the species abundance (Marr et al. 1992). P. lima concentrations showed a significant relationship between $P$. oceanica and the water column $\left(R^{2}=0.79\right)$, suggesting that the species, being a weekly swimming dinoflagellate that can even be affected by low water motion conditions (Richlen and Lobel 2011), might move from one compartment to another. 
The establishment of a direct relationship between the concentration of this species in the water column and on macrophytes allows us to assess the concentration in one compartment by referring to the concentration in the other one. Moreover, to the best of our knowledge, the toxicity threshold used for this species in the monitoring programmes was only established for the water column (Abdennadher 2014), so the use of this relationship to extrapolate to the substrata needs to be further investigated.

Ostreopsis cf. ovata had no preference for a given substratum, as indicated by the absence of a significant difference in concentrations between the substrata studied. The significant relationship found between the species on Padina and in the water column above this alga could be explained by the fact that this species is loosely attached to hard substrates and seaweeds with mucilaginous strands (Tindall and Morton 1998). Water motion could cause leaf agitation, allowing the shift of epiphytic species into the water column. A particularly

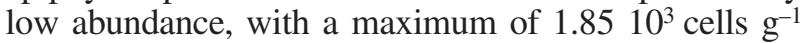
FW recorded on Posidonia leaves, was observed during this survey compared with the high species abundances observed in the western Mediterranean, where $7.210^{6}$ cells $\mathrm{g}^{-1} \mathrm{FW}$ was reported in Catalonia (Mangialajo et al. 2011), $2.510^{6}$ cells $\mathrm{g}^{-1} \mathrm{FW}$ on the Genoa coasts (Mangialajo et al. 2008) and $1.710^{6}$ cells $\mathrm{g}^{-1} \mathrm{FW}$ in the Adriatic Sea (Totti et al. 2010). In the eastern Mediterranean (Greece), a maximum abundance of $0.4110^{6}$ cells $\mathrm{g}^{-1} \mathrm{FW}$ was observed (Aligizaki and Nikolaidis 2006). These findings suggest that the study area might have some constraints preventing the accumulation of this toxic species, known to cause serious health concerns in other ecosystems and particularly in the Mediterranean (Totti et al. 2010, Cohu et al. 2011).

Leaves of the seagrass $P$. oceanica hosted the highest population, especially of $P$. lima, whereas Ostreopsis cf. ovata and other species were very scarce or planktonic (Fig. 3). This opposing pattern between Ostreopsis cf. ovata and P. lima was also illustrated in the divergence of these species in the cluster analysis (Fig. 4). A habitat separation between Ostreopsis spp. and Prorocentrum spp. has already been reported in the Pacific Ocean (Richlen and Lobel 2011). This behaviour could be attributed to allelopathic effects between dinoflagellates leading to possible niche separation. Indeed, some phytoplankton species, including $P$. lima (Sugg and VanDolah 1999), produce and release secondary metabolites that negatively affect the growth of other organisms (Rizvi and Rizvi 1992). These species quickly cause cell lyses of most competitors within minutes, when the latter are exposed to either certain amounts of the allelochemicals or to certain cell densities of the allelopathic algae. Such allelopathy is thought to reduce competition for nutrients, vitamins, etc. (Fistarol et al. 2004a). Indeed, the co-inertia plot showed that the distribution of Ostreopsis cf. ovata, and to a lesser degree $P$. lima, was explained by nitrogen, mainly nitrate and nitrite, which might suggest competition between these species for nitrogen availability. Both species were documented to be positively correlated with nutrient availability (nitrate, nitrite, phosphate, and silicate) concentrations in the waters surrounding Hawaii (Parsons and Preskitt 2007). Cohu et al. (2013) reported that phosphate concentration, rather than nitrogen or silicate concentration, was positively associated with Ostreopsis cf. ovata abundances in the north western Mediterranean Sea. Furthermore, many studies have shown that nutrient limitation decreases Ostreopsis cf. ovata growth, an effect that is more accentuated under N-limitation (Accoroni et al. 2014).

For $P$. oceanica, there is an increase in the cover of most epiphytic species in the apical and middle regions of the leaves (Fig. 5). This result had already been reported in previous studies (Alcoverro et al. 2004) and explained by the fact that the apical part of the leaves, and to a lesser degree the middle part, expose their epiphytes to high light intensities and water movement. This would promote photosynthetic organisms such as epiphytic macroalgae, which increase the nutrient intake from water and remove inhibitory substances (Trautman and Borowitzka 1999). Moreover, the epiphytic species zonation on leaves of Posidonia was reported to be related to the concentration of phenolic compounds produced in abundant quantities, depending on the state of stress caused by environmental conditions (Dumay et al. 2004). However, the use of artificial leaves made of plastic tape showed the same apico-basal distribution of epiphytic algae (Trautman and Borowitzka 1999), supporting the hypothesis that epiphyte settlement was unlikely to be the result of changes in the surface chemistry of the leaves (Borowitzka et al. 2006). These variations were likely due to differences in hydrodynamic or light intensity related to the shape and orientation of the leaves. The inner surface of adult and intermediate leaves seemed to be the most exposed (Borowitzka and Lethbridge 1989).

The concentration of $P$. lima on the outer surface of the Posidonia leaf, explained by the behaviour it uses to escape predators (Ben Brahim et al. 2010), is in opposition to the general behaviour of other epiphytic species, particularly Ostreopsis cf. ovata, which has been shown to prefer the inner face of Posidonia leaves (Alcoverro et al. 2004, Peirano et al. 2011). This would suggest competition for space between Ostreopsis cf. ovata and $P$. lima, and might support their apparently opposed distribution pattern.

\section{CONCLUSIONS}

This study has highlighted the diversity of epiphytic microorganisms on vegetated ecosystems, particularly on macroalgae, and has confirmed the previous finding on the potential of $P$. oceanica to accumulate epiphytic biomass. This finding suggests that more attention should be paid to the protection of the $P$. oceanica meadows and their associated epiphytes.

$P$. lima, by far the most abundant epiphytic toxic species on all vegetated substrates, showed a preference for $P$. oceanica. A significant correlation was found between the species concentration on that substrate and in the water column. More effort should be made to accurately determine this relationship under different hydrological conditions. One of the practical 
implications of this result is the recommendation to include the sampling of $P$. lima on Posidonia leaves in $\mathrm{HAB}$ monitoring programme and to set up the toxicity threshold of this species on $P$. oceanica leaves.

$P$. lima showed an opposed distribution pattern to that of Ostreopsis cf. ovata on Posidonia leaves, suggesting that competition for space and nutrient between the two species is likely. This hypothesis needs to be investigated in order to assess and apprehend the proliferation mechanisms of the two species.

\section{ACKNOWLEDGEMENTS}

We wish to thank Mr. Jamil JAOUA, founder and former head of the English Teaching Unit at the Sfax Faculty of Science, for proofreading our paper.

\section{REFERENCES}

Abdennadher M. 2014. Étude taxonomique et écophysiologique des dinoflagellés toxiques du Golfe de Gabès: Alexandrium minutum, Prorocentrum lima, Coolia spp. and Ostreopsis ovata. Ph.D. thesis. Univ. Science. Sfax, Tunisia.

Accoroni S., Romagnoli T., Pichierri S., et al. 2014. New insights on the life cycle stages of the toxic benthic dinoflagellate Ostreopsis cf. ovata. Harmful Algae 34: 7-16. https://doi.org/10.1016/j.hal.2014.02.003

Accoroni S., Romagnoli T., Pichierri S., et al. 2016a. Effects of the bloom of harmful benthic dinoflagellate Ostreopsis cf. ovata on the microphytobenthos community in the northern Adriatic Sea. Harmful Algae 55: 179-190. https://doi.org/10.1016/j.hal.2016.03.003

Accoroni S., Romagnoli T., Penna A., et al. 2016b. Ostreopsis fattorussoi sp. nov. (Dinophyceae), a new benthic toxic Ostreopsis species from the eastern Mediterranean Sea. J. Phycol. 52: 1064-1084

https://doi.org/10.1111/jpy.12464

Aissaoui A. Amri Z Akrout F et al. 2014. Environmental factors and seasonal dynamics of Prorocentrum lima population in coastal waters of the Gulf of Tunis, South Mediterranean. Water. Environ. Res. 86: 2256-2270.

https://doi.org/10.2175/106143014X13975035526266

Alcoverro T., Perez M., Romero J. 2004. Importance of withinshoot epiphyte distribution for the carbon budget of seagrasses: the example of Posidonia oceanica. Bot. Mar. 47: 307-312. https://doi.org/10.1515/BOT.2004.036

Aligizaki K., Nikolaidis G. 2006. The presence of the potentially toxic genera Ostreopsis and Coolia (Dinophyceae) in the North Aegean Sea, Greece. Harmful Algae 5: 717-730. https://doi.org/10.1016/j.hal.2006.02.005

Aligizaki K., Nikolaidis G., Katikou P., et al. 2009. Potentially toxic epiphytic Prorocentrum (Dinophyceae) species in Greek coastal waters. Harmful Algae 8: 299-311. https://doi.org/10.1016/j.hal.2008.07.002

APHA. 1992. Standard methods for examination of water and waste water. APHA, AWWA. Washington, DC., USA.

Armi Z., Turki S., Trabelsi E., et al. 2010. First recorded proliferation of Coolia monotis (Meunier, 1919) in the North Lake of Tunis (Tunisia) correlation with environmental factors. Environ. Monit. Assess. 164: 423-433. https://doi.org/10.1007/s10661-009-0903-z

Ben Brahim M., Hamza A., Hannachi I., et al. 2010. Variability in the structure of epiphytic assemblages of Posidonia oceanica in relation to human interferences in the Gulf of Gabes, Tunisia. Mar. Environ. Res. 70: 411-421. https://doi.org/10.1016/j.marenvres.2010.08.005

Ben Brahim M., Hamza A., Ben Ismail S., et al. 2013. What factors drive seasonal variation of phytoplankton, protozoans and metazoans on leaves of Posidonia oceanica and in the water column along the coast of the Kerkennah Islands, Tunisia? Mar. Pollut. Bull. 71: 286-298. https://doi.org/10.1016/j.marpolbul.2013.01.024

Bomber J.W., Rubio M.G., Norris D.R. 1989. Epiphytism of dinoflagellates associated with the disease ciguatera: substrate specificity and nutrition. Phycologia 28: 360-368. https://doi.org/10.2216/10031-8884-28-3-360.1

Borowitzka M.A., Lethbridge R.C. 1989. Seagrass epiphytes. In: Larkum A.W.D., McComb A.J., Shepherd S.A. (eds), Biology of Seagrasses. Elsevier, Amsterdam, pp. 304-345.

Borowitzka M.A., Lavery P., Keulen M. 2006. Epiphytes of seagrasses. In: Larkum A.W.D., Orth R.J., Duarte C.M. (eds), Seagrasses: Biology, Ecology and Conservation. Springer, Dordrecht, pp. 441-461.

https://doi.org/10.1007/978-1-4020-2983-7 19

Calabretti C., Citterio S., Delaria M.A., et al. 2017. First record of two potentially toxic dinoflagellates in tide pools along the Sardinian coast. Biodiversity 18: 2-7. https://doi.org/10.1080/14888386.2017.1310058

Caye G., Meinesz A. 1985. Observations on the vegetative development, flowering and seeding of Cymodocea nodosa (Ucria) Ascherson on the Mediterranean coasts of France. Aquat. Bot. 22: 277-289. https://doi.org/10.1016/0304-3770(85)90005-1

Chessel D., Dufour A.B., Dray S., et al. 2012. Analysis of ecological data: Exploratory and euclidean methods in environmental sciences. R package version 1.5-1. http://CRAN.Rproject. org/package=ade4

Clarke K.R., Warwick R.M. 2001. Change in Marine Communities: An Approach to Statistical Analysis and Interpretation. 2nd Edition, PRIMER-E, Plymouth, 172 pp.

Cohu S., Thibaut T., Mangialajo L., et al. 2011. Occurrences of the toxic dinoflagellate Ostreopsis cf. ovata in relation with environmental factors in Monaco (NW Mediterranean). Mar. Pollut. Bull. 62: 2681-2691. https://doi.org/10.1016/j.marpolbul.2011.09.022

Cohu S., Mangialajo L., Thibaut T., et al. 2013. Proliferations of the toxic dinoflagellate Ostreopsis cf. ovata in relation to depth, biotic substrate and environmental factors in North Western Mediterranean Sea. Harmful Algae 24: 32-44. https://doi.org/10.1016/j.hal.2013.01.002

Dauby P. 1989. The stable carbon isotope ratios in benthic food webs of the gulf of Calvi, Corsica. Contin. Shelf. Res. 9: 181-195. https://doi.org/10.1016/0278-4343(89)90091-5

David H., Kromkamp J.C., Orive E. 2017. Relationship between strains of Coolia monotis (Dinophyceae) from the Atlantic Iberian Peninsula and their sampling sites. J. Exp. Mar. Biol. Ecol. 487: 59-67. https://doi.org/10.1016/j.jembe 2016.11.014

Dolédec S., Chessel D. 1994. Co-inertia analysis: an alternative method for studying species: environment relationships. Freshw. Biol. 31: 277-293. https://doi.org/10.1111/j.1365-2427.1994.tb01741.x

Dumay O., Costa J., Desjobert J.M., et al. 2004. Variations in the concentration of phenolic compounds in the seagrass Posidonia oceanica under conditions of competition. Phytochemistry 65: 3211-3220. https://doi.org/10.1016/j.phytochem.2004.09.003

Fistarol G.O., Legrand C., Selander E., et al. 2004a. Allelopathy in Alexandrium spp.: effect on a natural plankton community and on algal monocultures. Aquat. Microb. Ecol. 35: 45-56. https://doi.org/10.3354/ame035045

Foden J., Purdie D.A., Morris S., et al. 2005. Epiphytic abundance and toxicity of Prorocentrum lima populations in the Fleet Lagoon, UK. Harmful Algae 4: 1063-1074. https://doi.org/10.1016/j.hal.2005.03.004

Frontier S. 1973. Etude statistique de la dispersion du zooplancton. J. Exp. Mar. Biol. Ecol. 12: 229-262. https://doi.org/10.1016/0022-0981(73)90056-7

Gambi M.C., Lorenti M., Russo G.F., et al. 1992. Depth and seasonal distribution of some groups of the vagile fauna of the Posidonia oceanica leaf stratum: structural and trophic analyses. PSZN I. Mar. Ecol. 13: 17-39. https://doi.org/10.1111/j.1439-0485.1992.tb00337.x

Gestoso I., Olabarria C., Troncoso J.S. 2010. Variability of epifaunal assemblages associated with native and invasive macroalgae. Mar. Freshw. Res. 61: 724-731. https://doi.org/10.1071/MF09251

Gray C.A., Otway N.M., Laurenson F.A., et al. 1992. Distribution and abundance of marine fish larvae in relation to effluent plumes from sewage outfalls and depth of water. Mar. Biol. 113: 549-559. https://doi.org/10.1007/BF00349698

Hallegraeff G.M. 2010. Ocean climate change, phytoplankton community responses, and harmful algal blooms: a formidable predictive challenge. J. Phycol. 46: 220-235. 
https://doi.org/10.1111/j.1529-8817.2010.00815.x

Hallegraeff G.M., Bolch C.J.S., Huisman J.M., et al. 2010. Planktonic dinoflagellates. Algae of Australia phytoplankton of temperate coastal waters. CSIRO Publishing/ABRS. Melbourne, $145-212$.

Hauxwell J., Cebrian J., Furlong C., et al. 2001. Macroalgal canopies contribute to eelgrass (Zostera marina) decline in temperate estuarine ecosystems. Ecology 82: 1007-1022. https://doi.org/10.1890/0012-9658(2001)082[1007:MCCTEZ] 2.0. CO;2

Ingarao C., Lanciani G., Verri C., et al. 2009. First record of Prorocentrum lima (Dinophyceae) inside harbor areas and along the Abruzzo region coast, W Adriatic. Mar. Poll. Bull. 58: 596-600. https://doi.org/10.1016/j.marpolbul.2009.02.012

Johnson M.P., Edwards M., Bunker F., et al. 2005. Algal epiphytes of Zostera marina: Variation in assemblage structure from individual leaves to regional scale. Aquat. Bot. 82: 12-26. https://doi.org/10.1016/j.aquabot.2005.02.003

Kikuchi T., Peres J.M. 1977. Consumer ecology of seagrass beds In: McRoy C.P., Helfferich C. (ed.) Seagrass ecosystems: a scientific perspective. Marcel Dekker, New York. pp. 147-194.

Koch E.W., Ackerman J.D., Verduin J., et al. 2006. Fluid dynamics in seagrass ecology, In: Larkum A.W.D., Orth R.J., Duarte C.M. (eds), Seagrasses: Biology, Ecology and Conservation, Springer, Amsterdam, The Netherlands, pp. 193-225. https://doi.org/10.1007/978-1-4020-2983-7 8

Legendre P., Legendre L. 1998. Numerical ecology (2nd English Edn). Elsevier Science B.V., Amsterdam. 853 pp.

Levasseur M., Couture J.Y., Weise A.M., et al. 2003. Pelagic and epiphytic summer distributions of Prorocentrum lima and $P$. mexicanum at two mussel farms in the Gulf of St. Lawrence, Canada. Aquat. Microb. Ecol. 30: 283-293. https://doi.org/10.3354/ame030283

Lewis F.G. 1984. Distribution of macrobenthic crustaceans associated with Thalassia, Halodule, and bare sand substrata. Mar. Ecol. Prog. Ser. 19: 101-113. https://doi.org/10.3354/meps019101

Mabrouk L., Hamza A., Sahraoui H., et al. 2009. Caractéristique et phénologie de l'herbier de Posidonia oceanica (L.) Delile sur les cotes de Mahdia (région est de la Tunisie). Bull. Inst. Nat. Sci. Tech. Océan. Pêche Salammbô. 36: 139-148.

Mabrouk L., Hamza A., Ben Brahim M., et al. 2011. Temporal and depth distribution of microepiphytes on Posidonia oceanica (L.) Delile leaves in a meadow off Tunisia. Mar. Ecol. 32: 148-161. https://doi.org/10.1111/j.1439-0485.2011.00432.x

Mabrouk L., Ben Brahim M., Hamza A., et al. 2014. Temporal and spatial zonation of macroepiphytes on Posidonia oceanica (L.) Delile leaves in a meadow off Tunisia. Mar. Ecol. 36: 77-92. https://doi.org/10.1111/maec. 12118

Mangialajo L., Bertolotto R., Cattaneo-Vietti R., et al. 2008. The toxic benthic dinoflagellate Ostreopsis ovata: quantification of proliferation along the coastline of Genoa, Italy. Mar. Poll. Bull. 56: $1209-1214$ https://doi.org/10.1016/j.marpolbul.2008.02.028

Mangialajo L., Ganzin N., Accoroni S., et al. 2011. Trends in Ostreopsis proliferation along the Northern Mediterranean coasts. Toxicon 57: 408-420. https://doi.org/10.1016/j.toxicon.2010.11.019

Marr J.C., Jackson A.E., McLachlan J.L. 1992. Occurrence of Prorocentrum lima, a DSP toxin-producing species from the Atlantic coast of Canada. J. Appl. Phycol. 4: 17-24. https://doi.org/10.1007/BF00003956

Maso M., Garcés E. 2006. Harmful microalgae blooms (HAB); problematic and conditions that induce them. Mar. Pollut. Bull. 53: $620-630$. https://doi.org/10.1016/j.marpolbul.2006.08.006

Mazzella L., Buia M.C., Spinoccia L. 1994. Biodiversity of epiphytic diatom community on leaves of Posidonia oceanica. In: Marino D. and Montresor M. (eds), Proceedings of the 13th Diatom Symposium, Biopress, Bristol, UK.

Mirella P.C.V., Brendan P.K., Melanie J.B., et al. 2012. Epiphyte grazing enhances productivity of remnant seagrass patches. Aust. Ecol. 37: 885-892.

https://doi.org/10.1111/j.1442-9993.2011.02332.x
Nesti U., Piazzi L., Balata D. 2009. Variability in the structure of epiphytic assemblages of the Mediterranean seagrass Posidonia oceanica in relation to depth. Mar. Ecol. 30: 276-287. https://doi.org/10.1111/j.1439-0485.2008.00275.x

Pagliara P., Caroppo C. 2012. Toxicity assessment of Amphidinium carterae, Coolia cfr. monotis and Ostreopsis cfr. ovata (Dinophyta) isolated from the northern Ionian Sea (Mediterranean Sea). Toxicon 60: 1203-1214. https://doi.org/10.1016/j.toxicon.2012.08.005

Parsons M.L., Preskitt L.B. 2007. A survey of epiphytic dinoflagellates from the coastal waters of the island of Hawaii. Harmful Algae 6: 658-669. https://doi.org/10.1016/j.hal.2007.01.001

Peirano A., Cocito S., Banfi V., et al. 2011. Phenology of the Mediterranean seagrass Posidonia oceanica (L.) Delile: medium and long-term cycles and climate inferences. Aquat. Bot. 94: 77-92. https://doi.org/10.1016/j.aquabot.2010.11.007

Perez M., Garcia T., Invers O., et al. 2008. Physiological responses of the seagrass Posidonia oceanica as indicators of fish farm impact. Mar. Pollut. Bull. 56: 869-879. https://doi.org/10.1016/j.marpolbul.2008.02.001

Richlen M.L., Lobel P.S. 2011. Effects of depth, habitat, and water motion on the abundance and distribution of ciguatera dinoflagellates at Johnston Atoll, Pacific Ocean. Mar. Ecol. Prog. Ser. 421: 51-66 https://doi.org/10.3354/meps08854

Rizvi S.J.H., Rizvi V. 1992. Allelopathy: basic and applied aspect. Chapman \& Hall, London, $480 \mathrm{pp}$. https://doi.org/10.1007/978-94-011-2376-1

Romagnoli T., Bavestrello G., Cucchiari E., et al. 2007. Microalgal communities epibiontic on the marine hydroid Eudendrium racemosum in the Ligurian Sea, during an annual cycle. Mar. Biol. 151: 537-552. https://doi.org/10.1007/s00227-006-0487-x

R-Development Core Team. 2006. R: A language and environment for statistical computing. R Foundation for Statistical Computing, Vienna, Austria. http://www.R-project.org/

Sghaier Y., Zakhama-Sraieb R., Benamer I., et al. 2011. Occurrence of the seagrass Halophila stipulacea (Hydrocharitaceae) in the southern Mediterranean Sea. Bot. Mar. 54: 575-582. https://doi.org/10.1515/BOT.2011.061

Sugg L.M., VanDolah F.M. 1999. No evidence for an allelopathic role of okadaic acid among ciguatera-associated dinoflagellates. J. Phycol. 35: 93-103 https://doi.org/10.1046/j.1529-8817.1999.3510093 x

Tindall D.R., Morton S.L. 1998. Community dynamics and physiology of epiphytic/benthic dinoflagellates associated with ciguatera. In: Anderson D.M., Cembella A.D., Hallegraeff G.M. (eds), Physiological Ecology of Harmful Algal Blooms, NATO ASI Series G41. Springer-Verlag, Berlin. pp. 293-313.

Totti C., Poulin M., Romagnoli T., et al. 2009. Epiphytic diatom communities on intertidal seaweeds from Iceland. Polar Biol. 32: 1681-1691. https://doi.org/10.1007/s00300-009-0668-4

Totti C., Accoroni S., Cerino F., et al. 2010. Ostreopsis ovata bloom along the Conero Riviera (northern Adriatic Sea): relationships with environmental conditions and substrata. Harmful Algae 9: 233-239. https://doi.org/10.1016/j.hal.2009.10.006

Trautman D.A., Borowitzka M.A. 1999. Distribution of the epiphytic organisms on Posidonia australis and P. sinuosa, two seagrasses with differing leaf morphology. Mar. Ecol. Prog. Ser. 179: 215-229. https://doi.org/10.3354/meps 179215

Underwood A.J. 1997. Experiments in ecology. Their logical design and interpretation using analysis of variance. Cambridge University Press, Cambridge. 504 pp.

Utermohl H. 1958. Zur Vervollkommung der quantitativen Phytomicroorganisms-Methodik. Mitt. Int. Ver. Theor. Angew. Limnol. 9: 1-38.

Van Dolah F. 2000. Marine algal toxins: origins, health effects, and their increased occurrence. Env. Health Persp. 108 (Suppl. 1): 133-141. 